\title{
EDUARDO CASANOVA: SUS TEORÍAS Y MÉTODOS ARQUEOLÓGICOS PARA EL ESTUDIO DEL SECTOR NORTE DE QUEBRADA DE HUMAHUACA
}

\author{
Paola Silvia Ramundo *
}

Fecha de recepción: 12 de diciembre de 2020

Fecha de aceptación: 27 de abril de 2021

\section{RESUMEN}

Se analizan las teorías y métodos que Eduardo Casanova aplicó en sus trabajos de campo en el sector norte de la Quebrada de Humahuaca (entre el Angosto de Perchel y el nacimiento de la Quebrada de Humahuaca, Jujuy). Estas investigaciones se desarrollaron en los años treinta del siglo XX y abarcaron el estudio de los sitios Pukara de La Cueva, Pukara Morado, Pueblo Viejo del Morado, Pueblo Viejo de La Cueva, Coctaca, Pukara de Humahuaca y Pukara de Calete. En las publicaciones del autor sobre dichos sitios se plasma su visión respecto a la existencia de una "Cultura Humahuaca" con uniformidad cultural, planteo que perduró por años en otros investigadores del sector. Además, el análisis de dichas producciones nos permite evaluar las metodologías y técnicas arqueo-antropológicas aplicadas en aquella época. Los aspectos que aquí se discuten y analizan constituyen otro aporte al estudio del desarrollo de la historia de la arqueología del Noroeste argentino.

Palabras clave: Casanova - historia de la arqueología - teoría - método - Quebrada de Humahuaca

\section{EDUARDO CASANOVA: HIS THEORIES AND ARCHAEOLOGICAL METHODS \\ FOR THE STUDY OF THE NORTH SECTOR OF QUEBRADA DE HUMAHUACA}

\section{ABSTRACT}

The theories and methods used by Eduardo Casanova in his field work in the northern sector of the Humahuaca Gorge (between Angosto de Perchel and the source of the Humahuaca Gorge,

\footnotetext{
* Consejo Nacional de Investigaciones Científicas y Técnicas, Pontificia Universidad Católica Argentina, Instituto de Investigaciones de la Facultad de Ciencias Sociales / Universidad de Buenos Aires, Facultad de Filosofía y Letras, Departamento de Ciencias Antropológicas. E-mails: paolaramundo@ conicet.gov.ar; paolaramundo@uca.edu.ar
} 
Jujuy) are analyzed. These surveys took place in the 1930s and encompassed the following sites: Pukara de La Cueva, Pukara Morado, Pueblo Viejo del Morado, Pueblo Viejo de La Cueva, Coctaca, Pukara de Humahuaca, and Pukara de Calete. The author's vision regarding the existence of a culturally uniform "Humahuaca Culture" is reflected on what he published on these sites; I argue that this interpretation persisted for years in other researchers of the same area. In addition, by analyzing these productions we can evaluate the archaeo-anthropological methodologies and techniques used then. The aspects under discussion and analysis represent another contribution to the study of the development of the history of archeology in the Argentine Northwest.

Keywords: Casanova - history of archeology - theory - method - Humahuaca Gorge

\section{INTRODUCCIÓN}

El trabajo tiene por objetivo específico analizar las teorías y métodos que Eduardo Casanova elaboró y puso en práctica durante sus exploraciones arqueológicas desarrolladas en el sector norte de la Quebrada de Humahuaca (un área comprendida entre el Angosto de Perchel y el nacimiento de la Quebrada de Humahuaca, dentro de la provincia de Jujuy, República Argentina [Reboratti et al. 2003]), porque nos interesa reconstruir parte de la historia disciplinar de esta área donde trabajamos desde hace más de una década y porque la metodología aplicada por Casanova fue un aporte que queremos destacar.

Sus investigaciones se concretaron durante los años treinta del siglo XX, cuando la Escuela Histórico-Cultural europea se introducía y consolidaba en Argentina (Ramundo 2008, 2010). Dichas exploraciones incluyeron el estudio de los siguientes sitios arqueológicos: Pukara de La Cueva, Pukara Morado (más el Pueblo Viejo del Morado al pie del anterior) y Pueblo Viejo de La Cueva (sitios de la quebrada de La Cueva, subsidiaria de la Quebrada de Humahuaca), así como también Coctaca, Pukara de Humahuaca y Pukara de Calete. ${ }^{1}$

En las publicaciones sobre estos sitios -que analizaremos historiográficamente-, Casanova plasma su visión respecto a la existencia de una "Cultura Humahuaca" con homogeneidad o uniformidad cultural (Casanova 1933, 1934, 1936), y nosotros postulamos que ese planteo perduró por algunos años en otros investigadores del sector.

El análisis de sus producciones también nos lleva a evaluar las metodologías y técnicas arqueo-antropológicas que aplicó en aquella época, y estos aspectos de su obra nos permiten alcanzar el objetivo general que guía nuestra investigación: realizar otro aporte a la historia de la arqueología del Noroeste argentino (NOA), que se suma al que otros investigadores han realizado sobre este protagonista (Montenegro et al. 2011; Otero 2013, entre otros).

Para abordar lo mencionado este trabajo analizará: la obra de Casanova en el marco de la historia de la arqueología argentina; cómo fueron y por qué desarrolló las investigaciones en esos sitios del sector norte de la Quebrada de Humahuaca (enfatizando en la metodología y la teoría aplicada) y, finalmente, cuál fue su interpretación general del área, así como el impacto que tuvo en otros investigadores que continuaron la labor en el sector.

\section{EDUARDO CASANOVA: SU OBRA EN LA ARQUEOLOGÍA ARGENTINA}

Eduardo Casanova (1902-1977) -nacido en España y naturalizado argentino-, egresó en 1929 de la Facultad de Filosofía y Letras de la Universidad de Buenos Aires (FFyL-UBA) como Profesor de Enseñanza Secundaria y Especial en Historia. Fue miembro fundador y primer secretario de la Sociedad Argentina de Antropología (SAA) creada en 1936 (Podestá 2007). Tuvo el cargo de profesor titular de la cátedra de "Arqueología Americana", así como el de director de la 
"Sección Arqueología" y decano sustituto -entre 1966 y 1968- de la Alta Casa de Estudios donde se formó. También fue responsable de la cátedra de "Arqueología Americana" de la carrera de Historia en la Facultad de Historia y Letras de la Universidad del Salvador (USAL) desde 1959, donde se lo distinguió como profesor emérito en 1968. Se desempeñó como director ad-honorem del Instituto de Arqueología en dicha universidad, institución que funda en 1964 y preside hasta 1970, y espacio donde crea en 1964 el Boletín de la Asociación Amigos (para ver mayores detalles sobre su biografía [Ramundo 2007]). Se desempeñó como jefe de la "Sección de Arqueología" del Museo Nacional de Historia Natural de Buenos Aires (MNHN) y secretario de la "Sección de Yacimientos Arqueológicos y Paleontológicos del Ministerio de Justicia e Instrucción Pública" de la República Argentina. Formó parte de numerosas expediciones realizadas por dicho museo, así como también por el Museo Etnográfico de Buenos Aires (MET) de la FFYL-UBA, y dirigió las primeras exploraciones del Instituto de Arqueología de la USAL en Jujuy. Actuó como director del Museo del Pukara de Tilcara, dependiente de la FFYL-UBA, desde 1960 hasta su fallecimiento en 1977.

En lo referente exclusivamente a sus investigaciones en Jujuy, como discípulo de Salvador Debenedetti (1884-1930), continuó con las tareas de restauración del Pukara de Tilcara que había iniciado con su maestro (a su vez discípulo de Juan Bautista Ambrosetti [1865-1917]).

Su labor en el Pukara de Tilcara (Casanova 1950) y el proyecto de creación de un museo en esa localidad jujeña (Casanova 1971) han sido tan trascendentes que dicha institución lleva su nombre. Entre sus obras citamos textos que resaltan su investigación en esa provincia (Casanova 1930, 1933, 1934, 1936, 1937, 1942a, 1942b, 1942c, 1948, entre otras).

Fuera de su investigación en Jujuy, y sin desmerecer el trabajo realizado en otras provincias argentinas, queremos destacar que su trayectoria y experiencia de campo lo condujeron a publicar normativas para realizar descripciones de los sitios arqueológicos bajo una rigurosa metodología (Casanova 1965, 1966a, 1966b); temas sobre los que profundizaremos. No obstante, debemos destacar que fue el contexto disciplinar el que también motivó la publicación de estos últimos trabajos. Hablamos de los años sesenta del siglo XX, cuando la arqueología argentina reclamaba una sistematización u ordenamiento en la forma trabajar para lograr estatus científico, fruto de una etapa previa de crisis y cambios (entre 1948 y 1960), cuando se recibe la influencia de la Escuela Norteamericana de Antropología a través de investigadores estadounidenses que trabajaron aquí (por ej. Bennett, Bleiler y Sommer) y de arqueólogos argentinos formados allí que regresan al país (por ej. Alberto Rex González), así como también por el refuerzo de la influencia europea con el arribo de Osvaldo Menghin, entre otros investigadores (Ramundo 2008, 2010). La necesidad de ordenamiento a partir de 1960 se puede visualizar, entre distintas iniciativas, al considerar necesaria la celebración de una Convención Nacional de Antropología en 1964 y $1965^{2}$ para sistematizar o unificar la nomenclatura y metodología utilizada dentro de este campo científico y así lograr un estatuto lingüístico y metodológico propio (Ramundo 2008, 2010).

\section{LAS INVESTIGACIONES DE CASANOVA EN EL SECTOR NORTE DE LA QUEBRADA DE HUMAHUACA}

\section{Los sitios del sector norte}

Al comenzar el recorrido de Casanova en esta área, y siguiendo un sentido norte-sur, encontramos dentro de la quebrada de La Cueva cuatro sitios arqueológicos: Pukara de La Cueva, Pukara Morado más el Pueblo Viejo del Morado al pie y, finalmente, Pueblo Viejo de La Cueva (figura 1).

El Pukara de La Cueva o HUM.06 (conocido localmente como "Picacho") se encuentra en la confluencia del arroyo Pukara con el río La Cueva. Es un pukara de residencia permanente con 
alta concentración edilicia, posibles áreas de funciones específicas, sectores de descarte fuera del área doméstica y donde los espacios conectados mediante vías de circulación se articulan con las viviendas (Ramundo 2016a). Está fechado por radiocarbono entre el Período de los Desarrollos Regionales II $^{3}$ (540 \pm 60 años AP [LP-2268, hueso]; $670 \pm 25$ años AP [UGAM\#8561, carbón]; $549 \pm 30$ años AP [MTC-15600, hueso]; $561 \pm 32$ años AP [MTC-15601, hueso]; $520 \pm 40$ años AP [LP- 2258, carbón]) y el Período Incaico (450 \pm 40 años AP [LP-2531, carbón]; $460 \pm 40$ años AP [LP-2420, carbón]) (Ramundo 2012, 2016a).

El Pukara Morado o HUM.07a (conocido localmente como "La Vizcachera” o "El Chorro") se ubica $4 \mathrm{~km}$ al norte de HUM.06. Presenta pocas estructuras dispersas en la cumbre y restos de una muralla de más de 1,5 m protegen su único acceso. Lo consideramos una fortaleza incaica (Ramundo 2016b) por la presencia de un rasgo arquitectónico típico de ese momento (una escalinata elaborada con piedras canteadas), así como también por el tramo secundario del Qhapaq Nan que pasa por la base del sitio.

Al pie de HUM.07a se encuentra el Pueblo Viejo del Morado o HUM.07b (conocido localmente como "El Rodeo"). Posee un sector habitacional -actualmente destruido- con estructuras que presentaban restos humanos y ajuares con material cerámico, lítico, óseo y minero-metalúrgico (se trata del área estudiada por Casanova en 1931). Separado de ese sector determinamos la existencia de un área de necrópolis con tumbas en falsa bóveda, donde se encontraron restos humanos con deformación craneana de tipo tabular erecta (Ramundo et al. 2019).

El Pueblo Viejo de La Cueva o HUM.08 se ubica sobre la margen occidental de la quebrada de La Cueva, 7 km al norte de HUM.07b. Siguiendo a Basílico $(1992,2008)$ se trataría de un semiconglomerado localizado sobre piedemonte, con estructuras de planta rectangular y circular, paredes de pirca sin argamasa y silos circulares con falsa bóveda, acceso a recursos hídricos, y está rodeado por andenes y cuadros de cultivo. Desde hace varias décadas el sitio se encuentra intervenido por los saqueos, la remoción continua y la reutilización de sus estructuras.

Fuera de la quebrada de La Cueva y en dirección sur, el sitio arqueológico conocido como Coctaca (figura 1) se ubica $10 \mathrm{~km}$ al noroeste de la ciudad de Humahuaca, exactamente a los S23 8'38.62" y $065^{\circ} 17^{\prime} 7.09$ ”. Casanova (1934) aclara que se localiza a “dos leguas y media” al noroeste de Humahuaca y presenta interminables andenes o bacanales destinados al cultivo, junto a los cuales aparecen viviendas que forman un núcleo principal y varias más pequeñas diseminadas. Casanova (1936) define a Coctaca como un "pueblo viejo" junto a andenes de cultivo y en una hoyada o meseta, con presencia de viviendas dispersas o agrupadas irregularmente y sin murallas. Dicho sitio fue estudiado previa y posteriormente por otros investigadores (Ardissone 1928; Greslebin 1929; Gatto 1934; Suetta 1967 y múltiples trabajos de Albeck, entre ellos Albeck 2019).

Más al sur, el Pukara de Humahuaca se localizaba en la ciudad de Humahuaca (figura 1), 128 km al norte de San Salvador de Jujuy, y fue definido por Casanova (1936) como un "pucará", es decir un "pueblo fortificado que ocupa una situación estratégica en lo alto de un cerro que le brinda protección de sus acantilados, reforzados, en los lugares más accesible, por gruesas muralla que dificultan todo intento de asalto" (Casanova 1936:223-224). Este sitio fue estudiado también por otros investigadores (Gatto 1943; Márquez Miranda 1944; etc.)

Finalmente, el sitio más austral trabajado por él en este sector de la Quebrada de Humahuaca es el Pukara de Calete (figura 1). Ubicado a los S23¹5'56.19”' y O65²1'56.93”, en la confluencia del río de la quebrada de Calete y el río Grande, y que también fue considerado por Casanova como un "pucará". Este fue abordado posteriormente por otros investigadores (Nielsen 1996; Mancini 2008, entre otros).

Si bien Casanova (1936) menciona la existencia de más "yacimientos" en el sector norte de Quebrada de Humahuaca (Yavi Chico, Sansana, Cangrejillos, Ugsara, Chulín, Rodero, Colanzulí, Titiconte, entre otros), los resultados de sus investigaciones en estos sitios no se publicaron. 


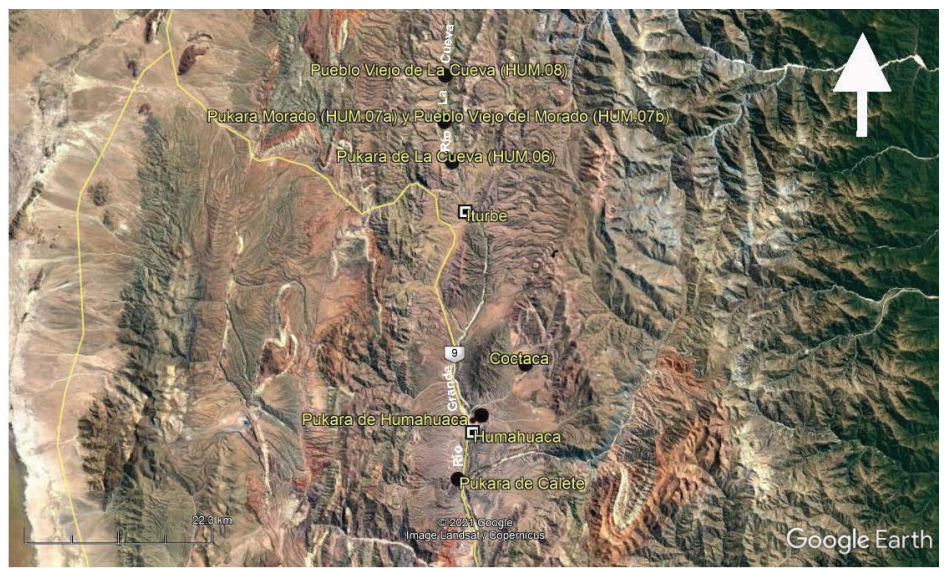

Figura 1. Mapa del sector norte de la Quebrada de Humahuaca con los sitios arqueológicos trabajados por Casanova, creado a partir de una imagen de Google Earth (2020)

Razones que condujeron a Casanova a trabajar los sitios del sector norte de Quebrada de Humahuaca

La quebrada de La Cueva

En 1931 la dirección del MNHN (actual Museo Argentino de Ciencias Naturales “Bernandino Rivadavia”) organizó una excursión con fines arqueológicos a la Quebrada de Humahuaca y regiones vecinas a cargo de Casanova -quien se desempeñaba como jefe de la "Sección Arqueología"-, y la asistencia de Santiago Gatto -que también trabajaba en el museo-. La expedición tuvo el apoyo del profesor Martín Doello Jurado (director de la institución), así como también contó con los avales: del Ministro de Justicia e Instrucción Pública, del interventor de la provincia de Jujuy, del presidente del Consejo de Educación de Jujuy, del juez federal de Jujuy y de un conocedor de la región, Milano Medenica. La meta era inspeccionar ruinas conocidas y localizar otras nuevas, así como también estudiar las condiciones en que podrían ser explotadas a futuro. De acuerdo a Casanova (1933) la tarea se realizó con premura porque disponía de diez días.

\section{Coctaca}

La misión oficial del MNHN de 1931 también desarrolló en Coctaca una “exploración preliminar de las ruinas prehistóricas, efectuando algunas excavaciones" (Casanova 1934:3), en el mismo viaje a la quebrada de La Cueva y con la dirección de Casanova y asistencia de Gatto (a pesar de que no lo aclara en Casanova [1934]). El objetivo fue realizar "una contribución que pueda servir de base a estudios de mayor amplitud" (Casanova 1934:3). Además, destaca que esto se debió a "los escasos medios de que disponíamos, así como también por la circunstancia de habernos tocado un verano extraordinariamente lluvioso que dificultó nuestras tareas" (Casanova 1934:3).

\section{Pukara de Humahuaca y Pukara de Calete}

Finalmente, con respecto a estos dos sitios, sabemos que Casanova los trabajó porque estudiamos los materiales que recuperó y que forman parte de las colecciones del MET, aunque 
jamás se publicaron los resultados. Solo en Casanova (1936) se aclara que el MNHN incluyó a la Quebrada de Humahuaca en su programa de investigaciones arqueológicas, del cual han surgido las publicaciones de Casanova $(1933,1934)$, pero nunca mencionó qué lo llevó al Pukara de Humahuaca o al Pukara de Calete, sitios a los que define como "pucarás". Las piezas de ambos sitios figuran en el catálogo del MNHN, fruto de la expedición de 1931 y, en Casanova (1933) se menciona que las investigaciones en esos pukaras se realizaron ese mismo año. Por lo tanto, las razones que lo condujeron a estos dos lugares serían las ya enunciadas.

\section{ENFOQUE TEÓRICO DE CASANOVA}

El esquema teórico que guio el problema de investigación de Casanova sobre la caracterización del pasado prehispánico de la Quebrada de Humahuaca y su sector norte, o dicho de otra manera ¿cómo enfrentó ese problema de investigación?, es algo que determinamos analizando dos elementos: 1) el contexto socio-histórico-disciplinar en el que desarrolló su trabajo; 2) las interpretaciones que presentó sobre la Quebrada de Humahuaca.

\section{El contexto disciplinar}

Desde los años treinta del siglo XX en adelante los enfoques históricos, surgidos en el Viejo Mundo y Norteamérica como consecuencia de la caída del marco Evolucionista Cultural, adquieren importancia en Argentina a partir de la influencia europea propiciada por el ambiente político (Ramundo 2005, 2008). La Escuela Histórico-Cultural europea se introdujo en Argentina con José Imbelloni en la década del treinta, porque este investigador (nacido en Italia) se trasladó en 1921 al país y contaba con estudios en antropología y ciencias naturales por los que obtuvo un doctorado en la Universidad de Padua. Aquí se desempeñó como profesor interino y luego como titular -desde 1939- en la cátedra de "Antropología" de la UBA y dentro de la USAL (misma institución donde trabajó Casanova) ejerció la docencia desde 1957 dictando "Antropología Cultural”. Dos hechos más vinculan ambas figuras: 1) fueron miembros fundadores y parte del Consejo Directivo de la SAA desde 1936 (Podestá 2007); 2) trabajaron en el MNHN, Imbelloni como jefe de la "Sección Antropología" entre 1931 y 1946 (Schobinger 1961) y, para la misma época, Casanova fue el jefe de la "Sección Arqueología" (Casanova 1933; Alfaro de Lanzone 1977).

Las ideas de Imbelloni se plasmaron en su obra Epítome de Culturología (1953 [1936]),

donde se reproducían las críticas más cáusticas del difusionismo al evolucionismo cultural, a la ideología del progreso y al racionalismo, a los que se consideraba teorías y principios puramente especulativos, exaltándose en cambio la idea romántica de la especificidad de cada cultura. La toma de posición, de carácter excluyente, conducía al extremo de intentar definir en forma agnóstica y empírica a cada cultura a través del 'patrimonio' de sus bienes o sea con un criterio de inventario, para lo cual se confiaba en la intuición histórica y morfológica (Madrazo 1985:30).

Para Madrazo (1985) la Escuela Histórico-Cultural se afianza debido al vacío teórico que el Evolucionismo Cultural había dejado y se trasforma en el único aporte significativo de teoría y método que se produjo en Argentina durante esa época, así como en un factor de discusión ideológica (Boschín y Llamazares 1984). Estas ideas histórico-culturales, por ejemplo, se hacen evidentes en los artículos presentados en el XXV Congreso Internacional de Americanistas realizado en La Plata (Argentina), donde el protagonista aquí analizado presentó uno de los textos que mencionamos (Casanova 1934). 


\section{Las interpretaciones de Casanova sobre la Quebrada de Humahuaca}

Considerando lo expuesto en el apartado anterior, Casanova podría considerarse un investigador de la Escuela Histórico-Cultural europea (Ramundo 2018b) debido a que constantemente se plantea discutir la inclusión de los sitios analizados del sector norte dentro de la Quebrada de Humahuaca a nivel cultural. Además, destaca conceptualmente que dichos sitios participan de los "caracteres" de la Quebrada, postulando la existencia de semejanzas entre los restos culturales (tipos de materiales y abundancia, etc.) de esos sitios con otros de la Quebrada troncal, así como -en menor medida- con sectores más lejanos, como el área calchaquí. Por ejemplo, en Casanova (1933) se destaca al comienzo y se reafirma al final que la quebrada de La Cueva es parte de la Quebrada de Humahuaca porque la considera su continuación natural y, por lo tanto, participa de los mismos caracteres. En esta línea argumental los materiales recuperados serán un elemento que utiliza para sostener esa postura. Algunos ejemplos son:

1) al describir unos vasos ornitomorfos del Pukara de La Cueva destaca que son semejantes a otros recuperados en la Isla de Tilcara (sitio del sector medio de la Quebrada de Humahuaca),

2) cuando habla sobre unas manos de mortero y dos conanas del sitio anterior las define semejantes a las del Pukara de Tilcara,

3) cuando detalla el material de Pueblo Viejo de La Cueva destaca que algunas cerámicas tienen "semejanzas (...) con las piezas extraídas de las ruinas de la quebrada de Humahuaca" (Casanova 1933:300) y otras son "exactamente iguales" a las encontradas en Coctaca y muy semejantes a otras de la Quebrada de Humahuaca, especialmente en el Pukara de Tilcara. Una pieza que describe como única para la quebrada de La Cueva, aclara que pertenece "al grupo de los llamados 'vasos pseudo-ápodos', de los que se han encontrado varios ejemplares en el pucará de Tilcara" (Casanova 1933:303). Sobre otras vasijas menciona su semejanza con vasos encontrados y descriptos por Debenedetti en la Isla de Tilcara, y que son abundantes en la Quebrada de Humahuaca,

4) cuando se refiere a los objetos de metal de Pueblo Viejo de La Cueva se plantea que un adorno representa un pájaro, cuya técnica de confección "es usual en la quebrada de Humahuaca" (Casanova 1933:316), como por ejemplo en La Isla de Tilcara.

La contundencia de su idea sobre la uniformidad cultural de la Quebrada de Humahuaca se afirma cuando dice que "Las industrias de la cerámica, de la piedra, del hueso, del tejido y del metal estaban grandemente desarrolladas y ofrecían las mismas técnicas que en toda la quebrada de Humahuaca" (Casanova 1933:318).

Consideramos importante resaltar además un dato sobre su interpretación de la quebrada de La Cueva que confirma la idea de la existencia de una cultura propia de "los humahuacas": "Como ejemplo de elementos extraños a la quebrada de Humahuaca propiamente dicha, podemos citar a los instrumentos de piedra, palas y azadones, que mostrarían el aporte de los pueblos situados al oeste y norte de los humahuacas" (Casanova 1933:318). Por lo tanto, la quebrada de La Cueva es la:

continuación geográfica de la de Humahuaca y los pueblos prehistóricos que la poblaron tuvieron el mismo patrimonio cultural; pero la situación de estos yacimientos de La Cueva, en el límite extremo de esta civilización, aparecen algunos elementos, cuya importancia y significado aún no es posible precisar, que pueden ser de gran valor para estudiar las relaciones de los humahuacas con otras tribus contemporáneas suyas (Casanova 1933:318).

En su publicación sobre Coctaca la idea de uniformidad cultural se ratifica: a) "Los objetos extraídos de las ruinas prehistóricas de Coctaca, a pesar de no ser abundantes ni ofrecer características especiales que los diferencien mayormente de los del resto de la zona de Humahuaca" 
(Casanova 1934:34); b) "Como en toda la quebrada de Humahuaca, el motivo más usado por los indígenas fue el reticulado, más o menos regular y con cuadrados de distintos tamaños" (Casanova 1934:36); c) "la llamada decoración 'en bandera o gallardete', que es tan peculiar en los grandes cántaros del pucará de Tilcara” (Casanova 1934: 37); d) "Los párvulos fueron depositados en urnas o en sepulcros redondos, pero no sería difícil que esto no fuera exclusivo y que, al igual que en pucará de Tilcara, se encuentren también inhumaciones directas de párvulos" (Casanova 1934:38).

Se suma una frase final y categórica cuando afirma: "El material arqueológico presenta gran variedad de formas y decoraciones, sin ofrecer rasgos esenciales que lo diferencien del hasta ahora conocido de la quebrada de Humahuaca" (Casanova 1934:38).

En su obra síntesis de la Quebrada de Humahuaca (Casanova 1936) todo lo dicho se reafirma. No se ocupa de las particularidades de los sitios publicados o no publicados, sino que sobre la base de sus estudios sintetiza la idea de una homogeneidad cultural en toda la extensión de la Quebrada de Humahuaca. Expresiones como "Cultura Humahuaca" o "foco principal de irradiación" (Casanova 1936:208) nos ayudan a confirmar lo dicho, así como también a sostener que su postura teórica es de impronta Histórico-Cultural. También lo destacan sus aseveraciones sobre: a) la necesidad de evaluar la posibilidad de "contactos de los humahuacas con las tribus chaqueñas y chichas" (Casanova 1936:209); b) la existencia de una "cultura típica y bastante uniforme que floreció en la quebrada y regiones anexas" (Casanova 1936:207), c) "El estudio de la civilización Humahuaca prueba que era un pueblo de cultura típicamente andina" (Casanova 1936:246), entre otras expresiones.

Su visión sobre la existencia de una "Cultura Humahuaca" con homogeneidad o uniformidad cultural previa a la llegada de los Incas (Casanova 1936), que lo lleva a "incluir indiscriminadamente todos los hallazgos en una cultura homogénea atribuida a los omaguacas históricos" (Nielsen 1997:19), fue consecuencia de "la aceptación de la postura de Boman sobre la poca profundidad temporal de las culturas del Noroeste argentino" (Nielsen 1997:18-19).

La idea sobre la homogeneidad de la "Cultura Humahuaca" perduró por algunos años en otros investigadores del sector norte de la Quebrada de Humahuaca. Así, por ejemplo, en Alicia Fernández Distel (1983) podemos apreciar la impronta de Casanova. Para la investigadora los sitios de la quebrada de La Cueva y Coctaca compartieron caracteres con la Quebrada de Humahuaca, sin embargo, no podemos afirmar lo mismo sobre los pukaras de Humahuaca y Calete porque la bibliografía que ella cita en su trabajo no es Casanova (1936), único texto donde el autor menciona ambos sitios. Interpretamos que no recurre a esa publicación porque es una síntesis de la Quebrada de Humahuaca y no un estudio detallado de los sitios. Respecto a la quebrada de La Cueva y Coctaca, Fernández Distel (1983) hace referencia a la materialidad, tipo de viviendas, localización de los hallazgos y la presencia de cerámica propia de otros sitios de la Quebrada de Humahuaca (como los estilos Isla Polícromo; Hornillos; Tilcara Negro sobre rojo). Esto se debe a que la información de base es tomada de Casanova o de textos que él cita. De todos modos, destacamos que el uso de ciertas expresiones y forma de trabajo por parte de Fernández Distel (1983) denotaría la presencia de diferentes marcos teórico-metodológicos: uno posiblemente cercano a la Escuela Histórico-Cultural Normativista al hablar de "Cultura Humahuaca" y al recurrir a extensas descripciones (algo propio de dicha corriente), otro donde apela al empleo de conceptos cronológicos aplicados en los enfoques de las corrientes norteamericanas de los años cincuenta y sesenta del siglo XX (por ej. Período Medio o Tardío), así como también utiliza una herramienta que le permite ordenar el panorama cerámico: los estilos propuestos por los investigadores norteamericanos Bennett, Bleiler y Sommer (1948).

Para la quebrada de La Cueva y Coctaca, al tomar la información de Casanova, establece que comparten caracteres con la Quebrada de Humahuaca y, por otra parte, esto la lleva a repetir datos brindados por Casanova (1933), que por estudios posteriores sabemos que son erróneos, por ejemplo, la altura y cantidad de recintos del Pukara Morado (Ramundo 2016b). 
En una publicación síntesis muy posterior (Fernández Distel 2007) reitera datos y conceptos de Casanova (1933) sobre el Pukara de La Cueva, Pukara Morado y Pueblo Viejo de La Cueva. Respecto a este último sitio no cita los estudios de Basílico $(1992,1994)$ que estaban publicados desde hacía más de una década. En el caso de Coctaca incluye más datos porque tiene en cuenta otros autores -discípulos de Casanova del Instituto de Arqueología de la USAL-, aunque cita a Casanova (1934) y su idea se mantiene al decir que el sitio "fue ocupado por la cultura Humahuaca" (Fernández Distel 2007:159). Para los ejemplos del Pukara de Calete y de Humahuaca tampoco recurre al texto de Casanova (1936), pero menciona autores contemporáneos que lo trabajaron, y el último sitio lo atribuye a la "Cultura Humahuaca".

Más allá de estas cuestiones, lo que deseamos rescatar es la posible impronta metodológica de Casanova sobre Fernández Distel. ${ }^{4}$ En este sentido, la investigadora arma un útil y detallado mapa arqueológico del departamento de Humahuaca que recupera justamente aquella información que -como veremos a continuación- pedía Casanova (1965), a lo que suma una serie de fichas resumen con los siguientes datos: nombre del sitio (más la asignación de un número), ubicación (aunque no se define la latitud y la longitud), zona geográfica, descripción, materiales presentes (o motivos, si se trata de arte rupestre), adscripción cultural, adscripción cronológica y, finalmente, la bibliografía disponible; información de gran importancia para quienes desarrollamos trabajos en este sector.

Susana Basílico es otra investigadora que presentó una postura semejante respecto a la homogeneidad cultural de los sitios del sector norte de la quebrada de Humahuaca. Cuando trabajó en la quebrada de La Cueva (Basílico 1992, 1994) apuntó a comparar el material que extrajo de HUM.08 con cerámica de estilo Isla, para establecer relaciones con sitios de la Quebrada de Humahuaca, aclarando que los elementos y estructuras de diseño tienen correspondencia con dicho estilo y, por lo tanto, de acuerdo a la autora, son Isla también. Destacó que la existencia de ese estilo en HUM.08 se debió a contactos inter-sitios que tuvieron estos pueblos. Basílico utiliza una concepción de estilo cerámico amplia y diferente a la de Bennett, Bleiler y Sommer (1948), donde estilo también involucra la producción, morfología, elementos y estructura del diseño, así como el análisis de pastas, visión que se podría relacionar con enfoques de corte procesual (corriente con fuerte impronta en Argentina durante los años ochenta del siglo XX, a pesar de que su introducción se puede rastrear desde la década del setenta en adelante [Ramundo 2008, 2010]).

Finalmente, solo queremos agregar que las investigaciones posteriores en la quebrada de La Cueva y su comparación con otros sitios del sector norte de Quebrada de Humahuaca (sitios que trabajan otros investigadores desde diferentes enfoques y que aquí no incluimos por falta de espacio) están a cargo nuestro desde hace mucho más de una década. Sin embargo, la postura teórica y la problemática de estudio cambiaron, ${ }^{5}$ mas no dejamos de reconocer el aporte metodológico realizado por Casanova -que detallaremos a continuación- para la sistematización de los sitios del área; fruto de aquel contexto disciplinar que condujo al desarrollo de la Primera Convención Nacional de Antropología, en la cual Casanova -como hemos mencionado- realizó sus aportes.

\section{METODOLOGÍA DE EDUARDO CASANOVA}

Como destacamos, promediando los años sesenta del siglo XX, el investigador publica unas normativas mínimas generales para la descripción y elaboración de un catálogo sistemático y mapa de yacimientos arqueológicos (Casanova 1965, 1966a, 1966b), fruto de la investigación realizada y de aquel contexto disciplinar particular que reclamaba una sistematización. Dado que a partir de 1960 surge una etapa dentro de la historia de la arqueología argentina donde se buscó desarrollar una disciplina más científica o profesional (Ramundo 2010). Esto se manifestó, entre otros factores, a través de la preocupación por la rigidez de conceptos y la necesidad de elaborar 
una terminología propia; fenómeno que se evidencia, como destacamos, en la celebración de la Primera Convención Nacional de Antropología realizada en 1964 y 1965 (Revista Etnía 1965; Pellisero 1969-70). Hablamos de ese espacio de discusión académico donde el investigador presentó un trabajo (Casanova 1965) para ser sometido a discusión y prueba, sensu el propio autor, y sobre el que reflexiona posteriormente (Casanova 1966a, 1966b). En estos textos enfatiza la necesidad de codificación en fichas de los sitios, tomando como ejemplo las aplicadas por John Rowe en Perú o las que utilizaba la Dirección Nacional de Estadísticas y Censos de Argentina a través de computadoras (Casanova 1966a). El objetivo fue establecer "reglas comunes que faciliten el trabajo de los Institutos que deseen colaborar en la confección de un Catálogo sistemático de los yacimientos arqueológicos argentinos" (Casanova 1965:1), las cuales presenta a modo de sugerencia. Así como también se buscaba que las "autoridades tomen las medidas indispensables para asegurar la protección, siquiera de los más importantes, de estos valiosos repositorios" (Casanova 1966b:8).

Los pasos procedimentales enumerados en Casanova (1965) y publicados en el contexto de la Convención Nacional donde intervinieron múltiples investigadores nacionales son: 1) distribuir las áreas o grupos de yacimientos; 2) elaborar fichas (estandarizadas) de cada uno de ellos; 3) intercambiar fichas entre instituciones para decir si acuerdan con estas o para que señalen sus discrepancias; 4) tareas complementarias: actualizar el fichero y pensar en preparar un "Mapa Arqueológico de la Argentina" (con el símbolo arqueológico correspondiente al tipo de yacimiento). Por otra parte, se enfatiza que las fichas descriptivas deberían incluir: nombre, ubicación, tipo, características, materiales, cultura, cronología, bibliografía, documentación y colecciones inéditas. Con respecto al nombre se recomendaba recuperar todos los conocidos, y en el caso de que fueran varios debía primar el criterio cronológico. La ubicación se debía marcar utilizando latitud y longitud, pero también era necesario indicar distancias a las instalaciones humanas más próximas. Sobre el tipo de yacimiento las categorías se establecieron con referencia a la experiencia de Casanova en el NOA (especialmente en la Quebrada de Humahuaca): "Pucará o Pueblo fortificado"; "Pueblo viejo o sin defensas"; "Andenes o instalaciones agrícolas"; "Cementerio"; "Paradero"; etc. (y en los yacimientos complejos se recomendó denominar primero con el rasgo predominante y luego los secundarios, por ej.: "pucará con cementerio y corrales"). Las características se debían indicar concisamente. Los materiales se presentarían de acuerdo al tipo (cerámica, piedra, madera, etc.), indicando número total de piezas y haciendo referencia a aquellos de capital importancia. En el caso de que el yacimiento tuviera varias capas culturales los materiales se presentarían capa por capa. También se aclaraba sobre el ítem cultura que era necesario mencionar las culturas y facies culturales representadas en el yacimiento (algo muy propio del enfoque Histórico-Cultural Normativista), destacando la necesidad de mencionar qué autor hacía determinada adscripción cultural, en el caso de existir divergencias al respecto. Con respecto a la cronología se pedía indicar la antigüedad adjudicada al yacimiento y sus capas arqueológicas -si las tuviera-, así como los métodos usados para calcularla. Sobre la bibliografía disponible de los sitios era necesario reflejar todas las publicaciones existentes, pero con un criterio peculiar: calificarlas en importancia con respecto al "conocimiento objetivo" que brindaban sobre el yacimiento (capital, mediana, escasa), algo subjetivo de determinar. El mismo criterio es el que propone para la documentación inédita (informes, notas, películas, series fotográficas, etc., que se encuentren en museos, bibliotecas, archivos u otros repositorios). Finalmente se destacaba mencionar las colecciones inéditas, aquellas depositadas en museos o colecciones privadas, pero no publicadas.

Nuestro análisis crítico de las obras de Casanova sobre el sector norte de la Quebrada de Humahuaca brinda otros elementos sobre la metodología y prácticas que implementó. Así, por ejemplo, en muchos casos apela y reitera una idea sobre aquello que lo conduce a explorar determinadas regiones. Siempre es "una contribución que pueda servir de base a estudios de mayor amplitud" (Casanova 1934:3) o bien es para inspeccionar ruinas conocidas y localizar 
otras nuevas, así como estudiar las condiciones en que podrían ser explotadas a futuro (Casanova 1933). Dicho de otro modo, los resultados publicados en la década del treinta tienen un "carácter preliminar" (Casanova 1934:38). Por lo tanto, podríamos plantear la existencia de una práctica metodológica reiterativa en aquellos trabajos del investigador, donde se realiza una labor muy preliminar y luego se publica aclarando que es necesario profundizar, aunque posteriormente él no lo concreta. Pero la particularidad (que nos da luz sobre el contexto socio-económico) es que siempre se destaca que aquello que no permite ahondar los análisis es la carencia de dinero y el escaso tiempo disponible. Sensu Casanova (1933) la tarea en la quebrada de La Cueva se realizó con premura porque disponía de diez días y contaba con recursos financieros "muy modestos" (Casanova 1933:235), algo que también destaca para el trabajo en Coctaca (Casanova 1934).

Otra cuestión metodológica llamativa es que en esa excursión de 1931 aclara que se trabajó poco por "habernos tocado un verano extraordinariamente lluvioso que dificultó nuestras tareas" (Casanova 1934:3). Esto genera una reflexión: ¿por qué realiza el trabajo durante el verano, cuando llueve en esa región? ¿Tendría algún impedimento para ir en otra etapa del año? ¿Lo obligaban a realizar esta misión oficial en esa fecha? Lo curioso es que Casanova sabía sobre este factor meteorológico, cuando aclara que Coctaca goza de un clima "con abundantes lluvias en verano" (Casanova 1934:28).

\section{Metodología aplicada en la quebrada de La Cueva}

Aquí Casanova también actuó como director de la excursión y Gatto se desempeñó como asistente. Junto con ellos se suma otro actor social, el señor Milano Medenica, un "conocedor de la región" que le revela la existencia de "ruinas arqueológicas en la quebrada de La Cueva" y actúa como "guía y compañero" (Casanova 1933:261). La figura de Medenica aparece mencionada en otro texto de Casanova (1930), cuando explora el cerro Morado en el Departamento de Iruya (Salta), donde se aclara que era "extranjero y culto". Más tarde, en una publicación de Márquez Miranda (1939) sobre arqueología de la provincia de Jujuy, se destaca que Medenica había trabajado previamente con Debenedetti y que también fue valuador de rentas, comisario de Iruya, su intendente en 1939 y administrador de la finca Santiago (Dayan 2003). Manifestaba una clara “devoción por la arqueología” (Márquez Miranda 1939:161), motivo por el cual se desempeñaba como comisario de yacimientos arqueológicos. Gracias a este cargo, "Debenedetti realizó excavaciones en Pueblo Viejo y Titiconte en Iruya; Casanova en Cerro Morado y en la Quebrada de La Cueva, en la zona de la finca de Iruya y la de Rodero y Negra Muerta, contando todos con la ayuda de Medenica" (Karasik 2008-09:215). Sobre este tema destacamos dos aspectos que nos ayudan a comprender parte de la metodología de Casanova. En primer lugar, la recurrencia en el uso de un mismo guía local para el trabajo de campo, a quien podríamos considerar un informante en términos antropológicos (Guber 2001). En segundo lugar, el hecho de que atribuya al propio Medenica la revelación de la existencia de los sitios de la quebrada de La Cueva, cuando -como ya explicamos (Ramundo 2018a)-, resulta curioso que Casanova no mencionara la existencia de una colección de la quebrada de La Cueva recuperada por el coleccionista Benjamín Muniz Barreto, que fuera obtenida en 1919 y contara con materiales procedentes del Pukara de La Cueva y Pueblo Viejo de La Cueva. Remarcamos esto porque "El Dr. Casanova en nuestro país, en una serie de conferencias dadas en la Facultad de Ingeniería de Buenos Aires, resaltó la importancia del conjunto arqueológico" (Sempé 1987:4), refiriéndose a la colección Muniz Barreto. Sin embargo, en su texto de 1933 no hace referencia a esa colección. Planteamos que esto podría deberse a un espíritu propio de la disciplina del momento, cuando los investigadores querían mostrar lo encontrado como algo prístino y descubierto solo por ellos. Aunque también podría ser consecuencia de cierto recelo a la labor previamente realizada, considerando especialmente que se ha 
planteado que Karl Schuel (enviado por Muniz Barreto a Jujuy y quien realiza las exploraciones en la quebrada de La Cueva durante 1919) se habría distanciado desde el año 1922 en adelante de Muniz Barreto y Debenedetti -con quien también trabajó- (Nielsen y Acevedo 2020), y recordemos que este último fue maestro de Casanova.

Si evaluamos lo propuesto por Casanova (1965), pero leyendo su texto de 1933, ya es posible perfilar algo del enfoque metodológico que presentó para el relevamiento de yacimientos. Así, por ejemplo, observamos con respecto a los nombres que menciona la existencia de los siguientes sitios en la quebrada de La Cueva: "el pucará de La Cueva, el pucará Morado y el Pueblo Viejo de La Cueva" (Casanova 1933:261), a los que suma el Pueblo Viejo del Morado, cuando describe el Pukara Morado y lo ubica al pie de este. Nunca destaca si se trata de los nombres que los lugareños dan a los sitios o si es él quien se los asigna. ${ }^{6}$ También habla de la existencia de otro posible sitio más al norte (Ojo de Agua) que no llega a explorar por falta de tiempo.

En lo referente a la ubicación de los sitios de la quebrada de La Cueva, ninguno de ellos es marcado utilizando latitud y longitud (ni en el texto ni el mapa adjunto, a diferencia de lo que ocurre con la publicación sobre Coctaca), pero sí se indican distancias a las instalaciones humanas más próximas. Así, por ejemplo, se aclara que "Remontando el río de La Cueva, a una distancia de dos leguas de Iturbe, se encuentra el pucará de La Cueva, ubicado en la margen derecha del río" (Casanova 1933:262). También se dice que "Desde el pucará de La Cueva remontamos el río unos 7 u 8 kilómetros en busca de otro yacimiento (...) el pucará Morado se encuentra en la margen derecha del río La Cueva, pero alejado del cauce del éste" (Casanova 1933:283). Finalmente menciona que Pueblo Viejo de La Cueva está "situado a una legua al norte del pucará Morado (...) sobre la margen derecha del río La Cueva" (Casanova 1933:295).

Respecto al tipo de yacimiento, Casanova (1933) define la existencia de dos pukaras (La Cueva y Morado) y dos pueblos viejos (Pueblo Viejo del Morado y Pueblo Viejo de La Cueva). Indica sus respectivas características de forma extensa y detallada, destacando los hallazgos de acuerdo al tipo de material (cerámica, piedra, madera, textil, metal, etc.). Allí aclara el número total de piezas y, sin mencionar las capas presentes en cada uno de los sitios, describe qué va encontrando a medida que excava. Menciona el tema de la cultura representada en los yacimientos, destacando que "La quebrada de La Cueva es la continuación geográfica de la de Humahuaca y los pueblos prehistóricos que la poblaron tuvieron el mismo patrimonio cultural" (Casanova 1933:318). En este trabajo no se hace alusión a la cronología o antigüedad adjudicada a los yacimientos, aunque se da por descontado que son contemporáneos a los sitios de la Quebrada de Humahuaca. También se menciona la bibliografía disponible de las exploraciones previas, específicamente Rosen (1924) y Boman (1908).

Si bien en Casanova (1965) se resalta la importancia de describir o al menos mencionar la existencia de colecciones inéditas de los sitios (como las colecciones privadas no publicadas), en su texto de 1933 no hace referencia a la colección de Muniz Barreto, con una importante cantidad de materiales procedentes de los sitios que Casanova excavó (Ramundo 2018a) y, tal como mencionamos, resulta difícil creer que desconociera su existencia, más cuando posiblemente haya trabajado con pobladores locales contemporáneos a la exploración de Schuel.

\section{Metodología aplicada en Coctaca}

Sobre la ubicación del sitio no se brinda latitud y longitud en el texto, pero sí aparecen esos datos en el mapa adjunto (figura 2), y también se indica la distancia a instalaciones humanas próximas cuando aclara que se encuentra a "dos leguas y media al noroeste de la ciudad de $\mathrm{Hu}$ mahuaca" (Casanova 1934:3). Por otra parte, destaca que "partiendo de este punto se remonta el pequeño río de Coctaca y pronto se nota, a derecha e izquierda, rastros de la antigua ocupación 
del hombre: interminables andenes o bancales destinados al cultivo" (Casanova 1934:3). También hace referencia al mencionado mapa, donde la denominación del sitio y la quebrada en que este se localiza aparece escrita como "Costaca". Por lo tanto, con respecto al nombre del sitio -al igual que en sus recomendaciones posteriores de 1965- utiliza todas las denominaciones conocidas.

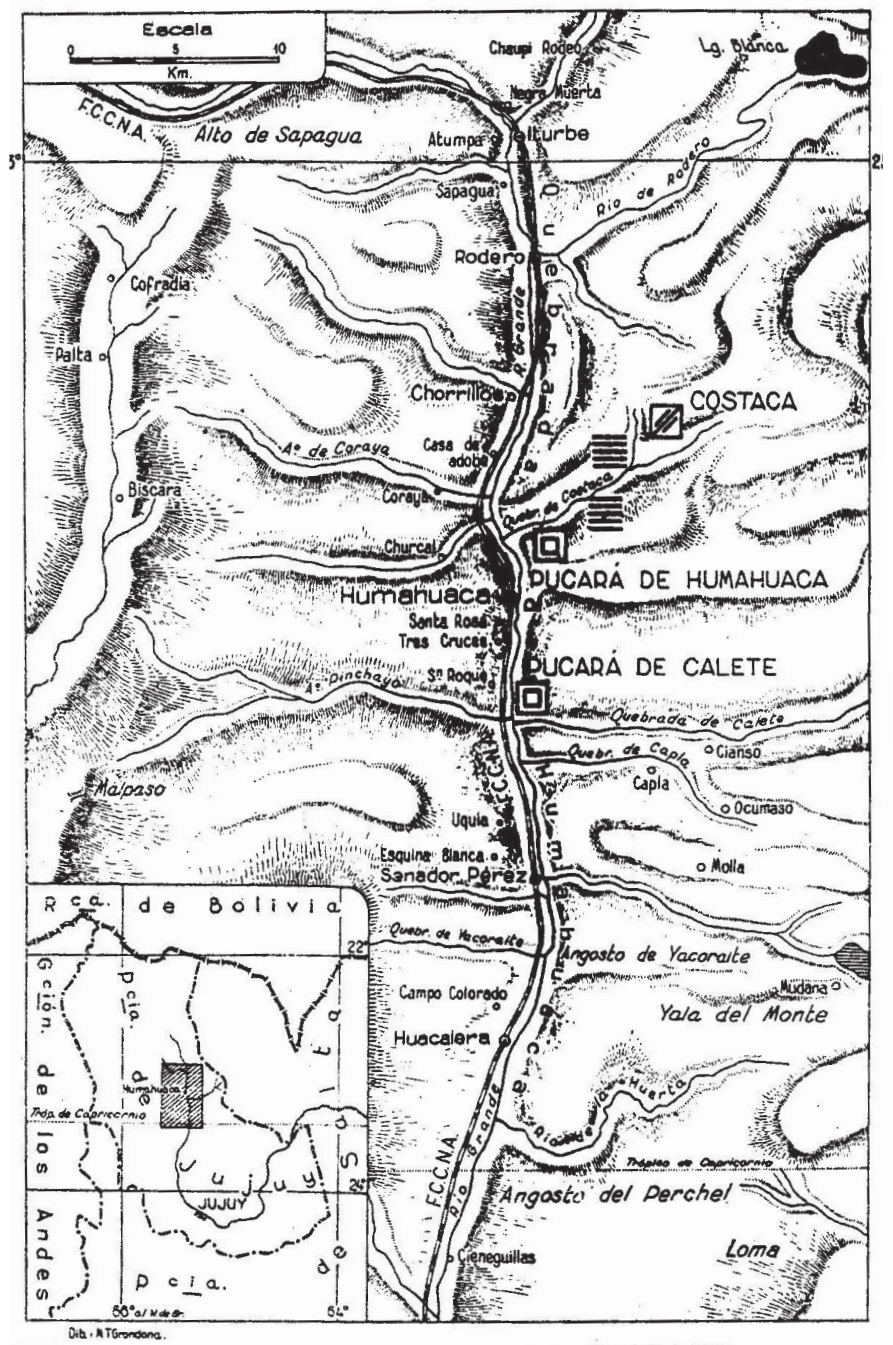

Figura 2. Mapa con la ubicación de Coctaca, publicado por Casanova (1934:26)

Define al yacimiento como "un pueblo dedicado principalmente a tareas agrícolas" (Casanova 1934:37). Se deduce -porque no lo dice en este texto, aunque sí en el trabajo de 1936- que estaríamos en presencia de un "pueblo viejo" al que se suman bancales o andenes de cultivo, acequias, graneros y sepulcros con restos humanos que menciona y describe. Destaca en reiteradas oportunidades que lo observado en el campo no es el poblado o núcleo principal, el cual podría "hallarse en algún abrigado rincón de una de las tantas quebradas que se comunican con la de Coctaca" (Casanova 1934:30) o "fuera de Coctaca" (Casanova 1934:38). Respecto a las características muestra los materiales recuperados de acuerdo al tipo (alfarería, piedra y hueso no humano), y si bien no indica el número total de piezas sí destaca el porcentaje relativo de los 
materiales de acuerdo a su importancia:

Predominan los objetos de barro cocido: siguen luego las piezas en piedra, y en mucho menor número las de hueso. En nuestras excavaciones no obtuvimos objetos de metal; en cuanto a los tejidos pudimos constatar su existencia, pero sin poder extraer ningún trozo que pudiera ser utilizado para estudios (Casanova 1934:34).

A pesar de esta detallada información no hace una presentación capa por capa, como propondrá en 1965.

Sobre la determinación de las culturas o facies culturales representadas no se hace una mención explícita, sin embargo, se aclara que "El material arqueológico presenta gran variedad de formas y decoraciones, sin ofrecer rasgos esenciales que lo diferencien del hasta ahora conocido de la Quebrada de Humahuaca" (Casanova 1934:38); por lo tanto, Coctaca pertenecería a la "Cultura Humahuaca". La cronología se podría definir mejor debido a que menciona -e ilustra en la figura 4 de la lámina III- la existencia de platos pato, ergología que es asignada a la esfera o presencia incaica en el NOA. Por otra parte, en la ilustración del material cerámico de la Lámina IV aparecen bordes de piezas con decoración "Humahuaca-Inca" (Ramundo 2017), por lo cual el sitio al menos podría adscribirse tentativamente al Período Incaico.

En lo referente a la bibliografía disponible se resalta la labor de Ardissone (1928), Greslebin (1929) y Gatto (1934), reflejando así las publicaciones disponibles en ese momento. También se hace referencia a la existencia de documentación inédita, se trata de los datos proporcionados a Debenedetti por parte de Benjamín Villafañe, "entusiasta por los estudios arqueológicos y por el pasado de Jujuy" [Casanova 1934:28]. En Casanova (1934) no se menciona la existencia de colecciones inéditas, las cuales no sabemos si realmente existieron, dado que en el caso de la quebrada de La Cueva existían, pero el investigador no las mencionó.

\section{Metodología aplicada en el Pukara de Humahuaca y Pukara de Calete}

Casanova no publica los resultados de las investigaciones de ambos sitios como lo hizo con los anteriores. Sin embargo, en Casanova (1936) presenta una síntesis de la Quebrada de Humahuaca donde brinda pinceladas de lo encontrado en ambos yacimientos. Inferimos que, como la campaña a esos sitios fue parte del mismo viaje, la metodología empleada ha sido análoga. La forma de registrar el material es idéntica a la realizada en los otros casos, porque en diciembre del 2019 estudiamos los materiales y registros de los pukaras de Humahuaca y Calete en el MET (los resultados de ese análisis serán presentados en otra publicación). Lo único que podemos destacar es que los nombres de ambos sitios, "Pucará de Humahuaca" y "Pucará de Calete", son mencionados de ese modo en Casanova $(1933,1936)$.

\section{REFLEXIONES FINALES}

En el presente artículo se analizaron dos aspectos de la producción de Eduardo Casanova sobre los sitios arqueológicos que excavó en el sector norte de la Quebrada de Humahuaca: su metodología y su postura teórica.

El Pukara de La Cueva, Pukara Morado, Pueblo Viejo del Morado, Pueblo Viejo de La Cueva, Coctaca, el Pukara de Humahuaca y el Pukara de Calete fueron su objeto de estudio en la década del treinta del siglo XX y, a partir de su experiencia en este sector de la Quebrada de Humahuaca (y posiblemente en otros del NOA que aquí no abordamos), Casanova fue forjando una metodo- 
logía de trabajo que dejó su impronta y que se preocupó por recuperar de forma detallada datos para las investigaciones arqueológicas: los nombres y tipos de sitios, su ubicación (especialmente a través de la creación de mapas), características principales, tipos de materiales, cronología, bibliografía disponible, documentación y colecciones inéditas. Ítems a relevar que luego plasma como un decálogo en otros textos donde destaca la importancia del registro exhaustivo de sitios (Casanova 1965, 1966a, 1966b), justamente en aquellos años sesenta del siglo XX, cuando la arqueología argentina lo reclamaba buscando mayor estatus científico.

A nivel teórico, bajo el marco de la Escuela Histórico-Cultural europea, postuló que la Quebrada de Humahuaca era homogénea culturalmente, aunque los estudios posteriores demostraron que no era necesariamente así (Nielsen 1996, 1997; Ramundo 2017; entre otros).

En el texto hemos mostrado que la impronta de la labor de Casanova -tanto metodológica como teórica- puede observarse en algunas generaciones posteriores. Luego, su visión sobre la homogeneidad cultural de la Quebrada de Humahuaca comenzó a cuestionarse; sin embargo, no podemos dejar de resaltar el valor metodológico de su sistematización de sitios para el estudio del área. Consideramos que estas directrices metodológicas del investigador fueron y siguen siendo valiosas para desarrollar nuestro trabajo, tanto en este sector del NOA como en cualquier parte del país.

Los cambios en las perspectivas teóricas a nivel local (iniciados a finales de la década del setenta y comienzos de los ochenta [Ramundo 2008]) y mundial (iniciados en los años sesenta) y, fundamentalmente, el dejar atrás el sello de las interpretaciones de Casanova, ha permitido evaluar con otro prisma la realidad del sector bajo estudio, preocupándonos por aquello que une a la Quebrada de Humahuaca pero también por todos los elementos y procesos sociales que distinguen a sus sectores y sitios.

Finalmente, queremos resaltar que el análisis crítico historiográfico aplicado sobre las producciones seleccionadas de Casanova nos permitió alcanzar nuestro objetivo, aquel que proponía aportar al estudio y comprensión del desarrollo de un parte de la historia de la arqueología argentina.

\section{AGRADECIMIENTOS}

A la Dra. Lorena Córdoba por la lectura del manuscrito y sus valiosos aportes, a la Dra. Mariela Zabala por facilitarme bibliografía, a la Mg. Luciana Sosa por el control del resumen en inglés, a los evaluadores por sus sugerencias y comentarios, sin embargo, todo lo escrito es de mi exclusiva responsabilidad. Esta investigación contó con el apoyo institucional del IICS-CONICET-UCA y el subsidio PIP 2015-2017-N¹1220150100217.

\section{NOTAS}

1 En este trabajo se utiliza la expresión "pukara" y no "pucará o pucara". Solo se emplearán estos últimos dos términos cuando los autores mencionados en el texto así los utilicen en sus citas textuales.

2 La Convención desarrolló su Primera Parte en Villa Carlos Paz (Córdoba) durante 1964, mientras la Segunda Parte fue en Resistencia (Chaco) en 1965.

3 El Período de los Desarrollos Regionales II o Tardío se extiende entre 1250 d.C. y 1430 d.C., y está precedido por el Período de los Desarrollos Regionales I o Temprano (entre 900 d.C. y 1250 d.C.) y le sigue el Período Incaico o Inka entre 1430 d.C. y 1536 d.C., de acuerdo a Nielsen y Boschi (2007).

4 La impronta también se podría observar en Raffino, quien publica un Atlas Arqueológico (Raffino 2007 [1988]) que incluye la mayoría de los sitios del NOA en distintos mapas con numeración correlativa y de acuerdo a diferentes períodos, describiendo características arquitectónicas, emplazamiento, función, bibliografía precedente, etc. 
5 Compartimos con Casanova que la quebrada de La Cueva "es parte integrante de la de Humahuaca de la cual es el escalón más elevado" (Casanova 1933:258), pero no acordamos con la idea de que esta "participa de todos los caracteres de la de Humahuaca" (Casanova 1933:258). Nuestra preocupación dejó de estar focalizada en la materialidad que permitía aseverar que la quebrada de La Cueva poseía idéntico patrimonio que la Quebrada de Humahuaca. Hoy también investigamos aquello que la vincula con otros sectores del NOA, así como también lo que la diferencia y le brindaba identidad propia. Nuestra premisa es que la quebrada de La Cueva (así como otros sitios del sector norte de Quebrada de Humahuaca) fueron parte de ese espacio, pero, puntualmente la quebrada de La Cueva, desarrolló elementos propios y se nutrió del aporte de otros sectores cercanos y lejanos como la Puna Jujeña, sur de Bolivia y norte de Chile, entre otros (Ramundo 2017).

6 Gracias a nuestros estudios recientes sabemos que Casanova no recuperó todos los nombres conocidos. Los sitios reciben desde antaño otras denominaciones por parte de los pobladores locales: Picacho, El Rodeo, El Chorro o la Vizcachera y Pueblo Viejo, respectivamente.

\section{BIBLIOGRAFÍA}

Albeck, M. E.

2019. Producción incaica en los Andes Centro Sur. Tecnología agrícola en Rodero y Coctaca, Argentina. Revista Española de Antropología Americana 49: 9-28.

Alfaro de Lanzone, L.

1977. Dr. Eduardo Casanova. Antiquitas XXIV-XXV: 1-8.

Ardissone, R.

1928. Coctaca. Gaea III(1): 1-65.

Basílico, S.

1992. Pueblo Viejo de La Cueva (Dpto. de Humahuaca, Jujuy). Resultado de las excavaciones en un sector del asentamiento. Cuadernos de la UNJU 3: 108-127.

1994. Análisis de las pastas de fragmentos de Pueblo Viejo de La Cueva y su correlación con la morfología y diseño pintado. En M. E. Albeck (ed.), Taller de Costa a Selva. Producción e Intercambio entre los Pueblos Agroalfareros de los Andes Centro Sur: 153-176. Tilcara, Instituto Interdisciplinario de Tilcara-Universidad de Buenos Aires.

2008. Las sociedades prehispánicas en la Quebrada de La Cueva (Humahuaca, Jujuy). Resultados preliminares sobre la ocupación del espacio. EnE. Cordeu (ed.), VICongreso Argentino de Americanistas, Tomo 2: 3-18. Buenos Aires, Dunken.

Bennett, W., E. Bleiler y F. Sommer

1948. Northwest Argentine Archaeology. New Haven, Yale University Press.

Boman, E.

[1908] 1997. Antigüedades de la Región Andina de la República Argentina y del Desierto de Atacama. San Salvador de Jujuy, EdiUNJU.

Boschín, M. y A. LLamazares

1984. La escuela histórico-cultural como factor retardatario del desarrollo científico de la arqueología argentina. Etnía 32: 101-151.

Casanova, E.

1930. Excursión arqueológica al cerro Morado (departamento de Iruya, provincia de Salta). Notas del Museo Etnográfico 3: 1-40.

1933. Tres ruinas indígenas en la Quebrada de la Cueva. Anales del Museo Argentino de Ciencias Naturales Bernardino Rivadavia 37: 255-320. 
Paola S. Ramundo - Eduardo Casanova: sus teorías y mÉtodos arqueológicos PARA EL ESTUdio del ...

1934. Observaciones preliminares sobre la arqueología de Coctaca (Provincia de Jujuy). Actas y Trabajos Científicos del XXV Congreso Internacional de Americanistas, Tomo I: 25-38. La Plata. Coni.

1936. La quebrada de Humahuaca. Historia de la Nación Argentina 1: 225-254.

1937. Contribución al estudio de la arqueología de La Isla. Relaciones I: 65-70.

1942a. El yacimiento arqueológico de Angosto Chico. Relaciones 3: 73-87.

1942b. El Pucará de Hornillos. Anales del Instituto de Etnografía Americana 3: 249-266.

1942c. Nota sobre el Pucará de Huichairas. Actas y Trabajos Científicos del XXV Congreso Internacional de Americanistas, Tomo II: 39-44. La Plata. Coni.

1948. Una estólica de la Puna Jujeña. Archivos Ethnos 2: 10-25.

1950. La Restauración del Pucará. Buenos Aires, Universidad de Buenos Aires.

1965. Normas mínimas generales para la descripción de yacimientos arqueológicos. Primera Convención

Nacional de Antropología (Segunda Parte): 1-4. Resistencia, Universidad Nacional del Nordeste.

1966a. Catálogo sistemático de yacimientos arqueológicos. Antiquitas 2: 10.

1966b. Catálogo sistemático de yacimientos arqueológicos II. Antiquitas 3: 8.

1971. El Museo Arqueológico de Tilcara (Antecedentes, funciones, guía). Publicación 2: 1-110.

Dayan, $\mathrm{P}$.

2003. ¿Soy un colla chiquitito? Construcción de la memoria en Iruya (Salta). Tesis de Licenciatura inédita,

Facultad de Filosofía y Letras, Universidad de Buenos Aires.

Fernández Distel, A.

1983. Mapa arqueológico de Humahuaca. Scripta Ethnologica Suplementa 4: 1-70.

2007. Diccionario Arqueológico. Jujuy, Secretaría de Turismo y Cultura de la Provincia de Jujuy.

Gatto, S.

1934. Un granero o silo en la quebrada de Coctaca (provincia de Jujuy). Actas y Trabajos Científicos del XXV Congreso Internacional de Americanistas, Tomo II: 51-56. La Plata, Coni.

1943. Ruinas del pucará de Humahuaca. Trabajo presentado en el Congreso de Historia Argentina del Norte y Centro. Córdoba, Argentina.

Greslebin, H.

1929. Tipo de cámara sepulcral en la quebrada de Coctaca (prov. de Jujuy). Physis IX: 327-334.

Guber, R.

2001. La Etnografía. Método, Campo y Reflexividad. Buenos Aires, Editorial Norma.

Imbelloni, J.

[1936] 1953. Epítome de Culturología. Buenos Aires, Nova.

Karasik, G.

2008-09. Haciendas, campesinos y antropología: conflictos sociales y colonialidad en el extremo noroeste argentino en la primera mitad del siglo XX. Travesía 10-11: 197-223.

Madrazo, G.

1985. Determinantes y orientaciones en la antropología argentina. Boletín del Instituto Interdisciplinario del Tilcara 1: 13-56.

Mancini, C. E.

2008. Metodología de la Arqueología de la Arquitectura «sin historia». Avances en la Quebrada de Calete, Jujuy. Trabajo presentado en el VI Congreso Argentino de Americanistas. Ciudad Autónoma de Buenos Aires, Argentina.

Márquez Miranda, F.

1939. Cuatro viajes de estudio al más remoto Noroeste Argentino. Revista del Museo de La Plata (Nueva Serie) I (6): 93-243. 
Relaciones de la Sociedad Argentina de Antropología 46 (1), enero-junio 2021: 57-75

1944. Excursiones de estudio realizadas durante el año 1944. Relaciones de viajes: Dos investigaciones en el pucará de Humahuaca (1933 y 1944). Revista del Museo de La Plata. Nueva Serie. Sección oficial 10(0): 123-141.

Montenegro, M., M. A. Aparicio, C. Otero y M. C. Rivolta

2011. Aportes de la Arqueología a la construcción de discursos sobre el pasado local. El caso del Pucará de Tilcara, Jujuy. Revista del Museo de Antropología 4: 81-88.

Nielsen, A.

1996. Demografía y cambio social en la quebrada de Humahuaca (Jujuy, Argentina), 700-1535 DC. Relaciones XXI: 307-354.

1997. Tiempo y Cultura Material en la Quebrada de Humahuaca 700-1650 d.C. Tilcara, Instituto Interdisciplinario Tilcara-Universidad de Buenos Aires.

Nielsen, A. y J. Acevedo

2020. Materiales arqueológicos de la Colección Schuel de Quebrada de Humahuaca (Jujuy, Argentina) en el National Museum of the American Indian (Smithsonian Institution). Revista del Museo de La Plata 5(1): 268-284.

Nielsen, A. y L. Boschi

2007. Celebrando con los Antepasados. Arqueología del Espacio Público en Los Amarillos. Quebrada de Humahuaca, Jujuy, Argentina. Buenos Aires, Mallku.

Otero, C.

2013. La Arqueología en el relato oficial del Estado Nacional. El caso del Pucará de Tilcara (Jujuy, Argentina). Arqueología Suramericana 6: 87-112.

Pellisero, N.

1969-70. Primera Convención Nacional de Antropología. Primera parte. Resoluciones. Provincia de Córdoba, Ciudad de Villa Carlos Paz. 24 al 29 de mayo de 1964. Comentario bibliográfico. Runa XII: 453-454.

Podestá, M.

2007. 70 años en la vida de la Sociedad Argentina de Antropología. Relaciones XXXII: 9-32.

Raffino, R.

2007. Poblaciones Indígenas en Argentina. Urbanismo y Proceso Social Precolombino. Buenos Aires, EMECE.

Ramundo, P.

2005. Historiografía de la Investigación Arqueológica Argentina, desde los Orígenes hasta Hoy. Tesis del DEA inédita, Facultad de Geografía e Historia, Universidad de Salamanca.

2007. Los aportes de los investigadores pioneros a la arqueología del Noroeste Argentino. Revista Temas de Historia Argentina y Americana 11: 179-217.

2008. Estudio Historiográfico de las Investigaciones sobre Cerámica Arqueológica en el Noroeste Argentino. Oxford, Archaeopress.

2010. Arqueología Argentina: una lectura arqueológica de su devenir histórico. Investigaciones y Ensayos 59: 469-510.

2012. Quebrada de La Cueva (Humahuaca, Jujuy): Cronología, espacialidad y cerámica arqueológica. Relaciones XXXVII(2): 329-354.

2016a. Perspectivas arqueológicas en la Quebrada de la Cueva (Depto. de Humahuaca, Jujuy). Anales de Arqueología y Etnología 70-71: 13-39.

2016b. Entre el cielo y la tierra. Pukara Morado de La Cueva o El Chorro, Departamento de Humahuaca, Provincia de Jujuy, Argentina. Anuario de Arqueología 8: 165-183.

2017. Consumption of Pottery in Quebrada de la Cueva Humahuaca, Jujuy. En A. Scaro, C. Otero y M. 
Paola S. Ramundo - Eduardo Casanova: sus teorías y mÉtodos arqueológicos PaRA EL ESTUdio del ...

B. Cremonte (eds.), Pre-Inca and Inca Pottery. Quebrada de Humahuaca, Argentina: 51- 80. Suiza, Springer.

2018a. El aporte del análisis de la Colección Muniz Barreto a los estudios de la quebrada de La Cueva, Humahuaca, Jujuy, Argentina. Mundo de Antes 12(1): 161-185.

2018b. Historia de las prácticas y producción de conocimiento arqueológico en la quebrada de La Cueva, Departamento de Humahuaca, Provincia de Jujuy. Trabajo presentado en IX Jornadas de Investigación en Antropología Social "Santiago Wallace". Buenos Aires, Argentina.

Ramundo, P., E. Gaál, C. Belotti, S. Julio, D. Sanz y M. Santos Giraldo

2019. Nuevo panorama sobre Pueblo Viejo del Morado (HUM.07b), Departamento de Humahuaca, Provincia de Jujuy. Estudios Sociales del NOA Nueva Serie 22: 37-65.

Reboratti, C., J. García Codrón, M. Albeck, H. Castro y M. Arzeno

2003. Una visión general de la Quebrada. En C. Reboratti (comp.), La Quebrada. Geografía, Historia y Ecología de la Quebrada de Humahuaca: 18-46. Buenos Aires, La Colmena.

Revista Etnía

1965. Comentario Bibliográfico sobre la Primera Convención Nacional de Antropología. Etnía 1: 28.

Rosen, E.

1924. Popular Account of Archaeological Research During the Swedish Chaco Cordillera Expedition. Stockholm, C. E. Fritze.

Schobinger, J.

1961. 75 Aniversario del Dr. José Imbelloni. Anales de Arqueología y Etnología XVI: 273-276.

Sempé, C.

1987. La colección Benjamín Muniz Barreto del Museo de La Plata. Novedades del Museo de La Plata 1(11): 92-93.

Suetta, J. M.

1967. Construcciones agrícolas prehispánicas en Coctaca (Provincia de Jujuy). Antiquitas 4: 1-9. 
